\title{
Interações medicamentosas com anti-hipertensivos
}

\author{
Drug interactions with antihypertensives \\ Interacciones medicamentosas con antihipertensivos
}

Recebido: 07/01/2022 | Revisado: 12/01/2022 | Aceito: 15/01/2022 | Publicado: 17/01/2022

\author{
Katiucce Borges de Morais \\ ORCID: https://orcid.org/0000-0002-0023-323X \\ Faculdade Unibras de Goiás, Brasil \\ E-mail: katiuccebm@gmail.com \\ Luciana Arantes Dantas \\ ORCID: https://orcid.org/0000-0001-8138-4824 \\ Faculdade Unibras de Goiás, Brasil \\ E-mail: luciana.dantas@unibras.digital \\ Celiana Maria Ferrarini Triches \\ ORCID: https://orcid.org/0000-0002-8031-3837 \\ Faculdade Unibras de Goiás, Brasil \\ E-mail: celianaferrarini@hotmail.com \\ Cínthia Alves Porfiro \\ ORCID: https://orcid.org/0000-0002-4398-0323 \\ Faculdade Unibras de Goiás, Brasil \\ E-mail: cinthiaporfiro@ hotmail.com \\ Manoel Aguiar Neto Filho \\ ORCID: https://orcid.org/0000-0002-3545-8746 \\ Faculdade Unibras de Goiás, Brasil \\ E-mail: manuel-aguiar@hotmail.com \\ Jacqueline da Silva Guimarães dos Santos \\ ORCID: https://orcid.org/0000-0002-0993-1114 \\ Faculdade Unibras de Goiás, Brasil \\ E-mail: guimaraes.js@alumni.usp.br
}

\section{Resumo}

A interação medicamentosa (IM) ocorre principalmente devido automedicação, polifarmácia ou erros de prescrição. Indivíduos hipertensos geralmente necessitam mais de um medicamento no tratamento, aumentando a probabilidade de possíveis IM proporcionais a quantidade de medicamentos utilizados, podendo levar a diferentes consequências como alterações no efeito farmacológico, reações adversas, toxicidade acumulativa, comorbidades ou morte. A classe terapêutica anti-hipertensivos, que se subdivide em diversas classes farmacológicas com o mesmo uso terapêutico, é a classe que mais se destaca em IM e fármacos anti-hipertensivos de classes farmacológicas diferentes podem interagir entre si e com outras classes de medicamentos em geral. Assim, o objetivo deste trabalho foi analisar estatisticamente as principais classes farmacológicas e os principais fármacos relacionados a IM com anti-hipertensivos por meio de uma revisão sistemática de literatura selecionando trabalhos publicados nos últimos vinte anos. As interações medicamentosas foram avaliadas segundo a gravidade, classificadas em leves, moderadas e graves. As classes farmacológicas causadoras de IM com anti-hipertensivos mais frequentes foram os hipoglicemiantes, antiinflamatórios não esteroides (AINES), diuréticos e combinação de dois tipos diferentes de anti-hipertensivos. Já os fármacos anti-hipertensivos que se destacaram em IM foram captopril, enalapril, hidroclorotiazida, propranolol, atenolol, anlodipino, amiodarona, losartana, espironolactona e furosemida. Portanto, os profissionais da área da saúde precisam se atentar para reações adversas oriundas dessas potenciais IM, notificar e intervir quando necessário para melhorar a eficiência do tratamento farmacológico evitando prejuízos à saúde.

Palavras-chave: Interações medicamentosas; Interações farmacológicas; Fármacos anti-hipertensivos; Drogas antihipertensivas; Medicamentos anti-hipertensivos.

\begin{abstract}
Drug interaction (DI) occurs mainly due to self-medication, polypharmacy or prescription errors. Hypertensive individuals usually need more than one drug for treatment, increasing the probability of possible DI proportional to the amount of drugs used, which can lead to different consequences such as changes in the pharmacological effect, adverse reactions, cumulative toxicity, comorbidities or death. The antihypertensive therapeutic class, which is subdivided into several pharmacological classes with the same therapeutic use, is the class that most stands out in DI and antihypertensive drugs from different pharmacological classes can interact with each other and with other classes of medications in general. Thus, the aim of this study was to statistically analyze the main pharmacological classes and the main drugs related to DI with antihypertensive drugs through a systematic literature review selecting works
\end{abstract}


published in the last twenty years. Drug interactions were evaluated according to severity, classified as mild, moderate and severe. The pharmacological classes causing DI with the most frequent antihypertensive drugs were hypoglycemic agents, non-steroidal anti-inflammatory drugs (NSAIDs), diuretics and a combination of two different types of antihypertensive drugs. The antihypertensive drugs that stood out in DI were captopril, enalapril, hydrochlorothiazide, propranolol, atenolol, amlodipine, amiodarone, losartan, spironolactone and furosemide. Therefore, health professionals need to pay attention to adverse reactions arising from these potential MI, notify and intervene when necessary to improve the efficiency of pharmacological treatment, avoiding damage to health.

Keywords: Drug interactions; Pharmacological interactions; Antihypertensive drugs; Antihypertensive medicines.

\begin{abstract}
Resumen
La interacción farmacológica (IF) se produce principalmente debido a errores de automedicación, polifarmacia o prescripción. Los hipertensos suelen necesitar más de un fármaco para su tratamiento, aumentando la probabilidad de un posible IF proporcional a la cantidad de fármacos utilizados, lo que puede conllevar diferentes consecuencias como cambios en el efecto farmacológico, reacciones adversas, toxicidad acumulada, comorbilidades o muerte. La clase terapéutica antihipertensiva, que se subdivide en varias clases farmacológicas con el mismo uso terapéutico, es la clase que más destaca en el IF y los fármacos antihipertensivos de diferentes clases farmacológicas pueden interactuar entre sí y con otras clases de medicamentos en general. Así, el objetivo de este estudio fue analizar estadísticamente las principales clases farmacológicas y los principales fármacos relacionados con el IF con fármacos antihipertensivos mediante una revisión sistemática de la literatura seleccionando trabajos publicados en los últimos veinte años. Las interacciones medicamentosas se evaluaron según la gravedad, clasificadas en leves, moderadas y graves. Las clases farmacológicas causantes de IF con los antihipertensivos más frecuentes fueron los hipoglucemiantes, los antiinflamatorios no esteroideos (AINE), los diuréticos y la combinación de dos tipos diferentes de antihipertensivos. Los antihipertensivos que destacaron en el IF fueron captopril, enalapril, hidroclorotiazida, propranolol, atenolol, amlodipino, amiodarona, losartán, espironolactona y furosemida. Por tanto, los profesionales sanitarios deben estar atentos a las reacciones adversas derivadas de estos potenciales IF, notificar e intervenir cuando sea necesario para mejorar la eficacia del tratamiento farmacológico, evitando daños a la salud.
\end{abstract}

Palabras clave: Interacciones con las drogas; Interacciones farmacológicas; Fármacos antihipertensivos; Drogas antihipertensivas; Medicamentos antihipertensivos.

\title{
1. Introdução
}

A pressão arterial é um dos elementos essenciais para o bom funcionamento do organismo, a qual é classificada em dois tipos, sistólica e diastólica. A pressão arterial sistólica ou considerada pressão arterial máxima, pode ser identificada no instante em que o ventrículo esquerdo bombeia sangue para a artéria aorta exercendo pressão geralmente entre 120 a 140 mmHg, conforme os padrões considerados normais. Já a pressão arterial diastólica ou pressão arterial mínima, é identificada no instante em que o ventrículo esquerdo volta a encher-se de sangue para reiniciar o processo da circulação sanguínea e, seu valor normalmente está dentro da média dos $80 \mathrm{mmHg}$ (Brasil, 2012; Malachias et al., 2016).

\subsection{Hipertensão Arterial Sistêmica (HAS)}

HAS é uma doença crônica, degenerativa e não transmissível cuja origem está relacionada a diversos fatores, como, herança genética, estresse, má alimentação, sedentarismo, entre outros, podendo apresentar modificações funcionais e/ou estruturais dos órgãos alvo, como: coração, encéfalo, rins e vasos sanguíneos. É representada por valores elevados e conservados de pressão arterial (PA) maior que 140 x $90 \mathrm{mmHg}$, podendo esse aumento ser isolado para pressão sistólica ou para pressão diastólica, e ainda estar associada a alterações metabólicas com altas chances de desencadear eventos cardiovasculares letais e não letais (Carvalho et al., 2017; Giles et al., 2009; Luzet al., 2018; Malachias et al., 2016; Silva et al., 2014).

Fava et al. (2017), Luz et al. (2018) consideram a HAS um grave problema de saúde pública devido alta porcentagem de internações e elevados custos médicos e assistenciais para o governo. Além disso, é principal fator de risco para doenças cardiovasculares e complicações como acidente vascular cerebral, infarto agudo do miocárdio, doenças renais crônicas, insuficiência cardíaca, doença arterial coronariana. Diversos fatores, como, obesidade, tabagismo, ingestão de álcool, sedentarismo e maus hábitos alimentares podem elevar a chance de surgimento da hipertensão arterial. 
O controle da HAS é feito de forma medicamentosa e não medicamentosa de acordo com quadro clínico e condições de risco demonstradas pelo paciente. Entende-se de tratamento não medicamentoso, mudanças nos hábitos de vida como exercícios físicos e dieta. Já o tratamento medicamentoso, refere-se ao tratamento na forma de monoterapia ou associação de medicamentos que são prescritos de acordo com a idade, histórico familiar e lesão ao órgão-alvo (Fava et al., 2017; Leão et al., 2020).

\subsection{Tratamento Medicamentoso}

A classe terapêutica anti-hipertensivos é subdividida por várias classes farmacológicas e subclasses, cada uma com seu mecanismo de ação específico, sendo representadas por uma variedade de fármacos cujo objetivo é atingir ou manter uma pressão arterial abaixo de 140x90 mmHg, como: diuréticos (tiazídicos, diuréticos de alça), inibidores do sistema reninaangiotensina (inibidores da enzima conversora de angiotensina (IECA), bloqueadores dos receptores de angiotensina, inibidores diretos da renina), fármacos simpaticolíticos (betabloqueadores, agonistas alfa2-adrenérgicos, alfa1-bloqueadores, simpaticolíticos centrais), bloqueadores de canais de cálcio, vasodilatadores, entre outros (Eschenhagen, 2019).

Os medicamentos são instrumentos fundamentais para o tratamento, recuperação e manutenção da saúde. Eles são preparações farmacêuticas constituídos de um ou mais fármacos, com o intuito de produzir efeitos terapêuticos. Já o fármaco é uma substância de estrutura química definida que geram alterações bioquímicas e fisiológicas. Desse modo, é imprescindível o conhecimento sobre farmacologia de forma a entender o mecanismo de ação e as possíveis interações dessas substâncias (Ioris \& Bacchi, 2019).

O propósito de fazer o tratamento farmacológico com anti-hipertensivo é diminuir os níveis de pressão arterial relacionado a morbidade e mortalidade (Luz et al., 2018). A aferição de pressão arterial alterada consiste o principal motivo de consulta médica e os medicamentos anti-hipertensivos são os mais prescritos em todo o mundo (Dos Santos et al., 2012).

Quando somente mudanças de hábitos não ajudam no controle da pressão arterial, faz-se necessário adicionar a farmacoterapia. As primeiras opções de tratamento medicamentoso são fármacos das classes farmacológicas: diuréticos, betabloqueadores, inibidores da enzima conversora de angiotensina ou bloqueadores de canais de cálcio (Luz et al., 2018).

Alguns anti-hipertensivos não geram resultados esperados quando administrados de forma isolada, sendo necessário associação para intensificar a ação farmacológica, obtendo um efeito sinérgico ou sinergismo, que é um tipo positivo de interação medicamentosa quando utilizada de forma racional. Apesar de algumas associações melhorarem o controle da pressão arterial, pacientes com doenças cardiovasculares geralmente possuem outras comorbidades, sendo necessário a polifarmácia por longo período de tempo e isso, torna mais susceptível a interações medicamentosas prejudiciais (Campana et al., 2009; Moreno et al., 2007).

\subsubsection{Polifarmácia}

A polifarmácia é considerada quando há uso de cinco ou mais medicamentos (Secoli, 2010). Apesar desta prática ser útil para obter o efeito terapêutico sinérgico ou para terapia de comorbidades ela aumenta o risco de IM, que pode causar alterações farmacocinéticas ou farmacodinâmicas em um ou mais medicamentos usados podendo gerar reações adversas, intensificar ou bloquear o efeito do medicamento, além de colocar em risco o tratamento e a segurança do paciente (Andrade \& Souza, 2018; De Lima \& De Godoy, 2017; Gomes et al., 2019).

A quantidade de fármacos superior a três ou mais produtos serve como fator indicativo para revisão dos medicamentos, e espera-se que um quarto dessas pessoas, utilizem pelo menos, um de forma imprópria (Gontijo et al., 2012). Tornou-se muito comum pacientes com doenças crônicas consumindo diferentes medicamentos, sendo considerado atualmente a era da polifarmácia (Bibiana et al., 2019). 
O consumo de muitos medicamentos pode estar relacionado há alguns fatores, como: diversos receituários, incompreensão do paciente em relação ao tratamento medicamentoso, automedicação, falta de informação dos médicos e farmacêuticos que realizam o acompanhamento farmacoterapêutico. Estes fatores favorecem o surgimento de IM.

\subsection{Interação Medicamentosa (IM)}

IM é um evento clínico causado por alteração de efeito de um medicamento quando administrado previamente ou simultaneamente com outra substância, podendo ser outro medicamento, alimento, álcool ou outras substâncias químicas (Tavares et al., 2013). A associação de determinados medicamentos pode intensificar uma resposta farmacológica de forma positiva, como acontece no sinergismo, ou pode ocorrer uma interação negativa chamada de antagonismo. Nesse caso um medicamento pode interferir na ação de outro, ou ainda, provocar sérios danos à saúde (Andrade \& Souza, 2018; Bibiana et al., 2019; Ioris \& Bacchi, 2019; Gomes et al., 2019; Jacomini \& Silva, 2011; Zanetti et al., 2017).

O resultado da IM pode alterar de irrelevante a causar danos irreversíveis até possivelmente fatal. As IM podem colaborar com a piora do quadro clínico do paciente, como também, o aumento de medidas hospitalares e período de internação. E as chances de IM são proporcionais a quantidade de medicamentos usados, sendo $13 \%$ em pacientes que usam dois medicamentos e $85 \%$ em pacientes que usam mais de seis medicamentos (Scrignoli et al., 2016).

O uso concomitante de medicamentos para o tratamento de outras doenças crônicas aumenta a probabilidade de possíveis interações medicamentosas (Pirmohamed, 2010). O que pode levar a diferentes consequências como reações adversas, toxicidade cumulativa, comorbidades, e, às vezes, até a morte (Amaral \& Perassolo, 2012). Sendo relevante, o questionamento sobre interações medicamentosas quando se faz associação de outros medicamentos com os anti-hipertensivos.

Existem dois tipos de IM terapêuticas, as farmacocinéticas e farmacodinâmicas (Andrade \& Souza, 2018). A primeira está relacionada as transformações na concentração de pelo menos um dos ativos envolvidos durante os processos de absorção, distribuição, biotransformação ou eliminação, afetando um dos ativos com modificações na concentração plasmática, tempo de meia vida, início de ação, entre outros (Lattuca et al., 2013; Ioris \& Bacchi, 2019). Tem possibilidade de acontecer modificação no processo de transferência do medicamento do local da administração para a corrente sanguínea, influenciando a concentração sérica de fármacos aplicada pelas vias orais, tópicas ou parenterais, excluindo somente a via intramuscular. E a demora da absorção do medicamento pode ser um fato clínico indesejável, principalmente em casos de sintomas agudos. Várias modificações podem ocorrer antes da absorção gastrointestinal. Desse modo, medicamentos que mudam a motilidade gastrintestinal podem elevar ou reduzir a velocidade de absorção se utilizados simultâneo (Tavares et al., 2013).

Já a IM farmacodinâmica refere-se à competição ou interferência dos ativos no mesmo sítio de ação, isto é, relacionado ao mesmo receptor ou enzima, que pode gerar sinergismo, potencializando o efeito ou antagonizando, que é o mesmo que promovendo efeito contrário (De Lima \& De Godoy, 2017; Gomes et al., 2019; Ioris \& Bacchi, 2019).

Existe também, outro tipo de IM não muito comum, que são as interações físico-químicas que podem ocorrer antes mesmo da sua administração, como, a combinação de dois medicamentos em uma seringa incompatíveis, gerando precipitação ou turvação da solução, mudança da cor do medicamento ou até ocasionando a inativação de um ou dos dois medicamentos envolvidos (Bibiana et al., 2019). Outros exemplos de interações físico-químicas são a foto-oxidação, pois a maioria dos medicamentos devem ser guardados ao abrigo de luz, e oxidorreduções resultantes da coadministração de alimentos ou bebidas.

Assim, as principais consequências de uma interação medicamentosa são a ineficácia farmacoterapêutica e a manifestação de reações adversas, as quais podem comprometer o quadro clínico do paciente (Carreira et al., 2020).

Segundo Bertollo et al. (2014), existem vários elementos que colaboram para o aumento de IM, como: o uso de medicamentos com alta ligação a proteínas plasmáticas, administração de medicamentos em pacientes com doenças crônicas, 
baixo índice terapêutico de alguns fármacos, a polifarmacoterapia, uso de medicamentos de venda liberada, consumo de chás e plantas medicinais e o uso crônico de medicamentos excretados lentamente pelo organismo.

De forma equivocada, julga-se que fitoterápicos ou plantas medicinais, por serem de origem natural, estão livres de substâncias químicas e reações colaterais. E por isso, as pessoas fazem o uso da automedicação, mas sem saber que esses compostos podem gerar reações boas ou ruins, como os alopáticos. Além disso, os indivíduos podem sofrer IM com outros fitoterápicos e os medicamentos industrializados. E essas interações podem ser de forma sinérgica, antagônica ou farmacocinéticas. Por isso, é arriscado usar medicamentos fitoterápicos sem orientação médica. Plantas não são inofensivas e nem podem ser de forma indiscriminada e nem associadas com medicamento prescrito pelo médico, até porque estudos já comprovaram que dependendo de como são usadas representam uma ameaça à saúde (Cardoso et al., 2009).

Segundo Cardoso et al. (2009), a maioria das pessoas não sabem que os chás, por ser natural e não passar por nenhum processo na indústria, está livre de elementos químicos e eventos adversos, contudo, muitos deles apresentam princípios ativos que geram efeito farmacológico e que pode causar reações no organismo, benéficas ou maléficas. Falta informações sobre chás, já que são de fácil acesso em supermercados e eles podem ser julgados como alimentos que por meio do processamento industrial já não possuem mais ação farmacológica. Todavia, os chás mantém seus ativos íntegros quando são preparados a partir da planta seca.

Existem interações entre medicamentos e alimentos que podem modificar a absorção gastrointestinais de ambos. E o medicamento pode gerar inativação do nutriente e/ou o alimento pode causar ineficácia terapêutica, presentando características físicas e bioquímicas próprias. O tempo de meia vida de muitos medicamentos pode ser reduzida quando a alimentação é prevalente em proteínas ou prolongada e rica em carboidratos (açúcar) lipídeos (gorduras). Os aminoácidos (constituintes das proteínas) elevam a atividade enzimática do sistema oxidase de função mista hepática, já os carboidratos e lipídeos reduzem a atividade. As interações entre medicamentos e alimentos podem ser relevantes quando houver a ineficácia terapêutica, aumento de eventos adversos do medicamento ou dificuldade na absorção de alimentos (Tavares et al., 2013).

Há interações desejáveis que promovem benefícios terapêuticos, como, a diminuição de eventos adversos, aumento da duração do efeito, potencializam a eficácia, diminuição da dose. E existe também, as indesejáveis que diminuem o efeito terapêutico ou promovem resultado contrário ao esperado, gera reações adversas e podem até fazer com que o estado do paciente piore (Tavares et al., 2013; Zanetti et al., 2017; Luz et al., 2018).

De acordo com alguns estudos as IM podem gerar maior custo financeiro para o indivíduo e para o sistema de saúde, como também, aumentar o tempo de internação em hospitais. Sendo necessário um acompanhamento rigoroso com revisão e otimização das prescrições para esse perfil de paciente (Bertollo et al., 2014; Gomes et al., 2019; Pinto et al., 2014; Rocha et al.,2017).

\subsubsection{Intensidade das IM}

O nível de resposta das IM pode ser classificada como: grave, moderada ou leve. Grave, pode levar a morte ou provocar danos permanentes. Moderada, gera piora do estado clínico do paciente necessitando tratamento adicional, internação ou aumento do período de internação. Leve, respostas podem ser importunas ou despercebidas, mas não afeta de forma considerável o objetivo da farmacoterapia e nem exige outros tratamentos (Leão et al., 2020; Matos et al., 2009).

Rocha, Alves e Vitorino (2017) observaram que na maior parte dos prontuários que alguns fatores, como, idade, presença de comorbidades, quantidade de medicamentos usados e quantidade de médicos que assistiam o paciente contribuíram para gerar IM, as quais foram na sua maioria moderada.

As consequências das reações moderadas podem provocar sérios danos ao estado fisiopatológico do paciente. Este tipo de interação requer tratamentos adicionais e maior período de internação muitas vezes. As IM classificadas moderadas não 
costumam apresentar grandes riscos aos pacientes. Contudo, não se pode relevar, pois elas podem piorar o quadro clínico do paciente ou até exigindo alteração no tratamento (Rocha et al., 2017; Tavares et al., 2013).

O objetivo deste trabalho foi realizar levantamento bibliográfico sobre as principais interações com medicamentos anti-hipertensivos. Descrever as principais interações medicamentosas com anti-hipertensivos; Relatar estatisticamente o percentual das principais interações medicamentosas com anti-hipertensivos; Listar os principais fármacos de cada classe farmacológica que podem prejudicar os pacientes hipertensos em seu tratamento, caso a interação seja estendida a classe inteira; Listar os fármacos responsáveis pela interação medicamentosa nos pacientes hipertensos, caso haja diferença de interação entre fármacos de uma mesma classe farmacológica.

\section{Metodologia}

Este trabalho é uma revisão sistemática de literatura, segundo Mattos (2015) sobre interações medicamentosas com anti-hipertensivos. A pesquisa bibliográfica foi realizada utilizando os descritores: "interações medicamentosas" e "interações medicamentosas AND anti-hipertensivos" utilizando aspas nas palavras compostas, operador booleano AND e o filtro somente no título nos portais Google Acadêmico e Portal Regional da BVS (Biblioteca Virtual em Saúde) que utilizam as bases de dados do Scientific Electronic Library Online (SCIELO), Literatura Latino-Americana e do Caribe em Ciências da Saúde (LILACS), Medical Literature Analysis and Retrieval System Online (MEDLINE) entre outras. Foram encontrados 1017 trabalhos publicados nos últimos vinte anos (2000-2020). Os critérios de inclusão na pesquisa foram trabalhos cujo foco principal estava relacionado ao tema deste trabalho, disponíveis na íntegra e nos idiomas português, espanhol ou inglês.

Após a exclusão de artigos duplicados e que não atenderam aos critérios de inclusão, foram selecionados 115 artigos para o trabalho de conclusão de curso. Destes, 45 foram utilizados na revisão sistemática de literatura, conforme Figura 1.

Figura 1. Detalhamento dos artigos incluídos na revisão

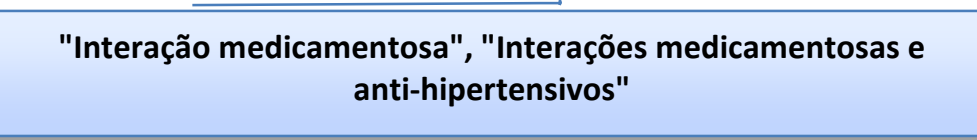

1017 - Estudos Encontrados

115 Estudos - Leitura dos Títulos e Resumos

Analise e Submissão de Critérios de Inclusão e Exclusão

45 Artigos Incluídos na Revisão Sistemática de Literatura

Fonte: Dados da Pesquisa (2020). 


\section{Resultados}

Foram selecionadas 45 publicações para esta revisão sistemática da literatura dos últimos 20 anos, 2000 a 2020, a distribuição quanto ao período lançado das fontes de pesquisas foram; 1 (2,27\%) de 2007, 2 (4,54\%) de 2008, 4 (9,09\%) de 2009, 2 (4,54\%) de 2010, 1 (2,27\%) de 2011, 6 (13,63\%) de 2012, 4 (9,09\%) de 2013, 3 (6,81\%) de 2014, 4 (9,09\%) de 2016, $3(6,81 \%)$ de 2017, 4 (9,09\%) de 2018, 8 (18,18\%) de 2019 e 2 (4,54\%) de 2020. Já em relação aos tipos de publicações, foram: revisão de literatura 12 (27,27\%), pesquisa de campo 27 (61,36\%), relato de casos 0 (0\%) trabalhos de conclusão de curso ou monografia $0(0 \%)$, dissertação $0(0 \%)$, tese $0(0 \%)$, livro $1(2,27 \%)$, outros $4(9,09 \%)$.

E dessas 45 publicações, 12 artigos de pesquisa, em relação ao período publicado, estão distribuídos: 2 (1,74\%) artigos são do ano de (2012), 1 (0,87\%) artigos em 2016, 1 (0,87\%) artigos em (2017), 3 (25,0\%) artigos em 2018, seguido de $4(3,47 \%)$ artigos em 2019 e por fim $1(0,87 \%)$ artigos em 2020.

Dos 12 artigos do tipo pesquisa de campo, verificou-se que a distribuição média entre os gêneros em porcentagem foi de 58,85\% ( $\pm 0,17)$ para o gênero masculino e 39,52\% $( \pm 0,11)$ para o gênero feminino, conforme Figura 2.

Figura 2: Distribuição de gênero dos artigos de pesquisa de campo.

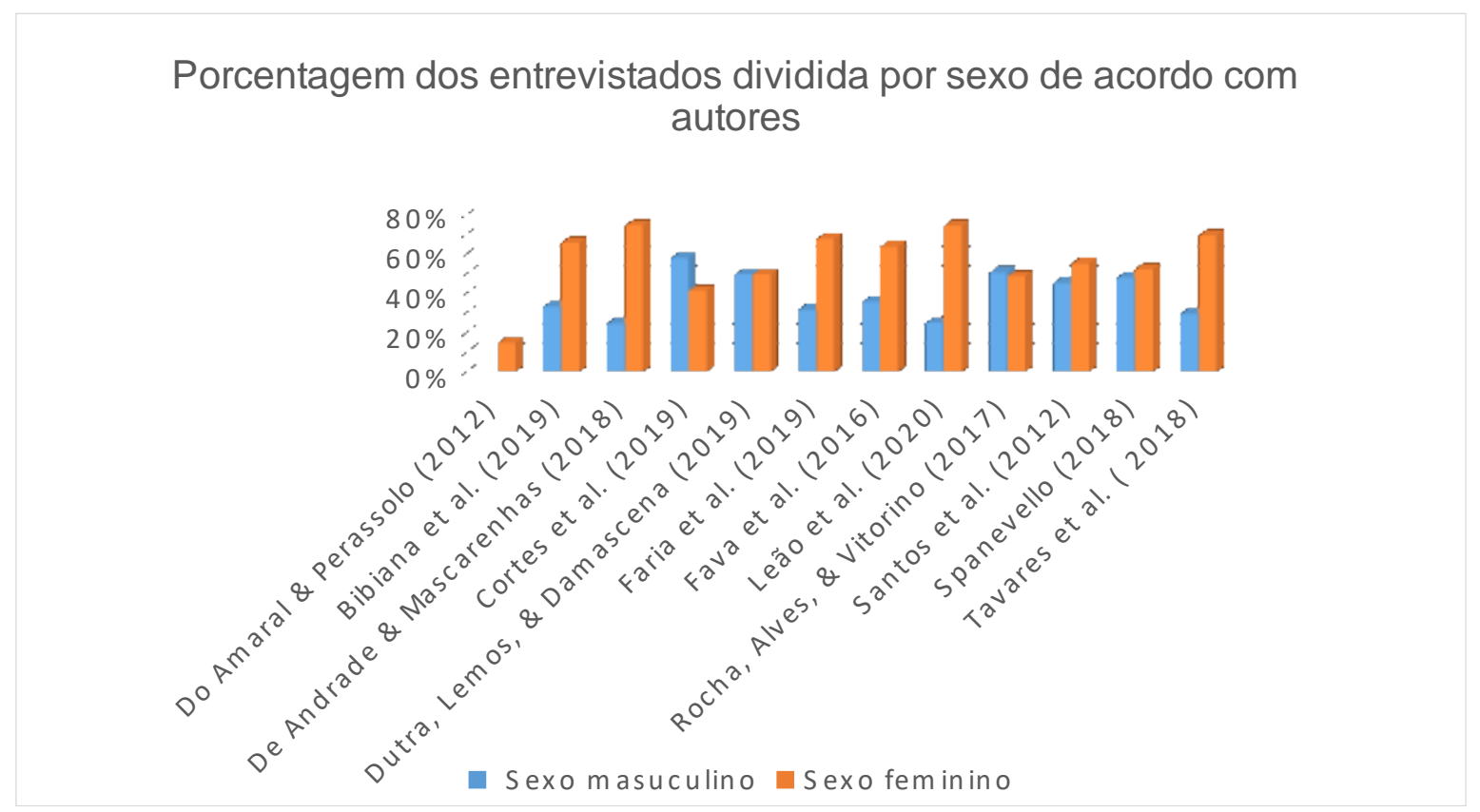

Fonte: Dados da Pesquisa (2020).

As interações medicamentosas foram avaliadas segundo a gravidade classificada em três níveis: leve, moderado e grave. Dentre as diferentes classes farmacológicas relatadas responsáveis por interação medicamentosa, foram avaliadas as IM com anti-hipertensivos, foco do nosso trabalho.

As médias proporcionais e desvios padrão (DP) de interação medicamentosa com anti-hipertensivos consideradas nível leve encontradas foram entre os fármacos apresentados na Tabela 1. 
Tabela 1: Interação medicamentosa considerado nível leve.

\begin{tabular}{ccl}
\hline Interação medicamentosa & Média e DP & Referências \\
\hline AAS X Captopril & $7,15 \% \pm 0,080$ & $\begin{array}{c}\text { Amaral \& Perassolo, 2012; Bibiana et al., 2019; De Andrade \& } \\
\text { Mascarenhas, 2018; Dos Santos et al., 2012; Tavares et al., 2013. }\end{array}$ \\
AAS x Hidroclorotiazida & $3,10 \% \pm 0,034$ & Amaral \& Perassolo, 2012; De Andrade \& Mascarenhas, 2018. \\
Hidroclorotiazida x Losartana & $1,70 \% \pm 0,014$ & Amaral \& Perassolo, 2012; Santos et al., 2012. \\
Levotiroxina x Propranolol & $0,38 \% \pm 0,004$ & Amaral \& Perassolo, 2012; Santos et al., 2012 \\
\hline
\end{tabular}

Siglas: AAS, ácido acetilsalicílico; DP, Desvio Padrão. Fonte: Dados da Pesquisa (2020).

A interação do AAS entre captopril e hidroclorotiazida, pode gerar redução do efeito do anti-hipertensivo. E a combinação entre hidroclorotiazida e losartana, pode acontecer redução adicional da pressão arterial. Já em relação a associação entre levotiroxina e propranolol, ocorre a diminuição da concentração do hormônio T3 (Tri-iodotironina) e diminuição da eficácia do propranolol.

As IM com anti-hipertensivos consideradas nível moderado foram separadas por grupos: IM entre diferentes classes de anti-hipertensivos (Tabela 2); IM entre anti-hipertensivos e hipoglicemiantes (Tabela 3); IM entre anti-hipertensivos e antiinflamatórios não esteroides (AINES) (Tabela 4); IM entre anti-hipertensivos e outras classes farmacológicas (Tabela 5).

As médias proporcionais de IM entre dois anti-hipertensivos de diferentes classes encontradas estão representadas na Tabela 2 . 
Tabela 2: Interação medicamentosa considerado nível moderado A: anti-hipertensivos x anti-hipertensivos.

\begin{tabular}{|c|c|c|}
\hline Interação medicamentosa & Média e DP & Referências \\
\hline Amiodarona x Losartana & $1,09 \% \pm 1,14$ & Bibiana et al., 2019; Dutra, Lemos, \& Damascena, 2019. \\
\hline Anlodipino x Atenolol & $0,59 \% \pm 0,14$ & Amaral \& Perassolo, 2012; Santos et al., 2012. \\
\hline Atenolol x Captopril & $3,26 \% \pm 3,24$ & Amaral \& Perassolo, 2012; Santos et al., 2012. \\
\hline Captopril x Digoxina & $13,29 \% \pm 15,85$ & Amaral \& Perassolo, 2012; Tavares et al., 2013. \\
\hline Captopril x Furosemida & $5,79 \% \pm 7,08$ & Amaral \& Perassolo, 2012; Bibiana et al., 2019; Santos et al., 2012. \\
\hline Captopril x Hidroclorotiazida & $8,05 \% \pm 5,29$ & $\begin{array}{l}\text { Amaral \& Perassolo, 2012; Bibiana et al., 2019; De Andrade \& } \\
\text { Mascarenhas, 2018; Santos et al., 2012; Tavares et al., } 2013 .\end{array}$ \\
\hline Captopril x Propranolol & $4,10 \% \pm 2,06$ & Amaral \& Perassolo, 2012; Santos et al., 2012 \\
\hline Digoxina x Furosemida & $7,10 \% \pm 11,62$ & $\begin{array}{l}\text { Amaral \& Perassolo, 2012; Bibiana et al., 2019; Santos et al., 2012; } \\
\text { Tavares et al., 2013. }\end{array}$ \\
\hline Enalapril x Digoxina & $19,44 \% \pm 24,55$ & Amaral \& Perassolo, 2012; Tavares et al., 2013. \\
\hline Enalapril x Furosemida & $15,39 \% \pm 12,42$ & Amaral \& Perassolo, 2012; Bibiana et al., 2019; Santos et al., 2012. \\
\hline Enalapril x Hidroclorotiazida & $6,70 \% \pm 7,06$ & $\begin{array}{l}\text { Bibiana et al., 2019; De Andrade \& Mascarenhas, 2018; Santos et al., } \\
\text { 2012; Tavares et al., } 2018 .\end{array}$ \\
\hline Furosemida x Propranolol & $0,56 \% \pm 0,36$ & Bibiana et al., 2019; Dutra et al., 2019; Santos et al., 2012. \\
\hline $\begin{array}{l}\text { Hidroclorotiazida } \mathrm{x} \\
\text { Propranolol }\end{array}$ & $3,85 \% \pm 2,08$ & Amaral \& Perassolo, 2012; Bibiana et al., 2019; Santos et al., 2012. \\
\hline Losartana x Espironolactona & $2,13 \% \pm 2,59$ & $\begin{array}{l}\text { Bibiana et al., 2019; Dutra et al., 2019; Rocha et al., 2017; Santos et al., } \\
2012 .\end{array}$ \\
\hline
\end{tabular}

Sigla: DP, Desvio Padrão. Fonte: Dados da Pesquisa (2020).

Combinação entre amiodarona e losartana pode aumentar os níveis plasmáticos de Losartana e diminuir os níveis plasmáticos do metabólito ativo. Podem ocorrer a diminuição da pressão arterial quando acontece as combinações entre os fármacos: anlodipino e atenolol, atenolol e captopril, enalapril e hidroclorotiazida, captopril e hidroclorotiazida, captopril e propranolol. Combinações como: captopril e digoxina e enalapril e digoxina, podem reduzir a excreção da digoxina em pacientes com disfunção renal. Pode causar em hipotensão postural (primeira dose) as associações entre furosemida com enalapril e ou captopril. Já a coadministração entre furosemida e propranolol pode gerar em hipotensão, bradicardia. E interação entre hidroclorotiazida e propranolol pode aumentar o risco de hiperglicemia. Já a hipertrigliceridemia e hidroclorotiazida e propranolol pode aumentar o risco de hipercalemia.

As médias proporcionais de IM entre anti-hipertensivos e hipoglicemiantes encontradas estão representadas na Tabela 3. 
Tabela 3: Interação medicamentosa considerado nível moderado B: anti-hipertensivos x hipoglicemiantes.

\begin{tabular}{|c|c|c|}
\hline Interação medicamentosa & Média e DP & Referências \\
\hline Atenolol X Glibenclamida & $1,31 \% \pm 0,013$ & Amaral \& Perassolo, 2012; Bibiana et al., 2019; Tavares et al., 2013. \\
\hline Atenolol X Insulina NPH & $2,7 \% \pm 0,0007$ & Amaral \& Perassolo, 2012; Bibiana et al., 2019. \\
\hline Captopril X Glibenclamida & $5,80 \% \pm 0,036$ & Amaral \& Perassolo, 2012; Bibiana et al., 2019; Tavares et al., 2013. \\
\hline Captopril X Metformina & $3,93 \% \pm 0,045$ & $\begin{array}{l}\text { Amaral \& Perassolo, 2012; Bibiana et al., 2019; De Andrade \& Mascarenhas, } \\
2018 .\end{array}$ \\
\hline Enalapril X Glibenclamida & $6,82 \% \pm 0,023$ & $\begin{array}{l}\text { Amaral \& Perassolo, 2012; Bibiana et al., 2019; Santos et al., 2012; Tavares et al., } \\
2018 .\end{array}$ \\
\hline Enalapril X Metformina & $8,21 \% \pm 0,049$ & $\begin{array}{l}\text { Amaral \& Perassolo, 2012; Bibiana et al., 2019; Tavares et al., 2013; Tavares et } \\
\text { al., } 2018 .\end{array}$ \\
\hline Enalapril X Insulina NPH & $8,43 \% \pm 0,074$ & Amaral \& Perassolo, 2012; Bibiana et al., 2019; Tavares et al., 2013. \\
\hline Glibenclamida X Hidroclorotiazida & $7,33 \% \pm 0,043$ & Amaral \& Perassolo, 2012; De Andrade \& Mascarenhas, 2018; Santos et al., 2012. \\
\hline Glibenclamida X Propranolol & $2,14 \% \pm 0,009$ & Amaral \& Perassolo, 2012; Bibiana et al., 2019. \\
\hline Insulina $\mathrm{x}$ Furosemida & $3,74 \% \pm 0,034$ & Amaral \& Perassolo, 2012; Dutra et al., 2019; Rocha et al., 2017. \\
\hline Insulina $\mathrm{x}$ metformina & $4,58 \% \pm 0,043$ & Amaral \& Perassolo, 2012; Dutra et al., 2019. \\
\hline Insulina NPH X Losartana & $1,73 \% \pm 0,018$ & Bibiana et al., 2019; Dutra et al., 2019. \\
\hline Insulina NPH X Propranolol & $1,96 \% \pm 0,011$ & Amaral \& Perassolo, 2012; Bibiana et al., 2019. \\
\hline Metformina X Hidroclorotiazida & $7,68 \% \pm 0,032$ & Amaral \& Perassolo, 2012; De Andrade \& Mascarenhas, 2018; Santos et al., 2012. \\
\hline Metformina X Propranolol & $3,02 \% \pm 0,009$ & Amaral \& Perassolo, 2012; Bibiana et al., 2019; Tavares et al., 2013. \\
\hline
\end{tabular}

Sigla: DP, Desvio Padrão. Fonte: Dados da Pesquisa (2020).

Combinações entre atenolol e glibenclamida, atenolol e insulina NPH, glibenclamida e propranolol pode gerar hipoglicemia ou hiperglicemia. Associações entre captopril e glibenclamida, captopril e metformina, enalapril e glibenclamida, enalapril e metformina podem levar ao aumento de hipoglicemia. Interações entre: glibenclamida e hidroclortiazida, insulina e furosemida, insulina e metformina, metformina e hidroclorotiazida podem reduzir o efeito hipoglicemiante.

As médias proporcionais de IM entre anti-hipertensivos e anti-inflamatórios não esteroides (AINES) encontradas estão representadas na Tabela 4. 
Tabela 4: Interação medicamentosa considerada nível moderado C: anti-hipertensivos x AINES.

\begin{tabular}{|c|c|c|}
\hline Interação medicamentosa & Média e DP & Referências \\
\hline AAS X Anlodipino & $10,0 \% \pm 0,098$ & De Lima \& Godoy, 2017; De Andrade \& Mascarenhas, 2018. \\
\hline AAS X Atenolol & $16,4 \% \pm 0,211$ & $\begin{array}{l}\text { Amaral \& Perassolo, 2012; Bibiana et al., 2019; De Lima \& Godoy, 2017; Neiva et al., } \\
\text { 2019; Santos et al., 2012; Tavares et al., 2018. }\end{array}$ \\
\hline AAS X Captopril & $4,12 \% \pm 0,049$ & Amaral \& Perassolo, 2012; Bibiana et al., 2019. \\
\hline AAS X Enalapril & $0,14 \%$ & Bibiana et al., 2019. \\
\hline AAS X Losartana & $3,84 \% \pm 0,024$ & De Andrade \& Mascarenhas, 2018; Faria et al., 2019; Santos et al., 2012. \\
\hline AAS X Nifedipino & $0,02 \%$ & De Andrade \& Mascarenhas, 2018. \\
\hline AAS X Propranolol & $4,02 \% \pm 0,034$ & $\begin{array}{l}\text { Amaral \& Perassolo, 2012; Bibiana et al., 2019; Tavares et al., 2013; Tavares et al., } \\
2018 .\end{array}$ \\
\hline Atenolol X Dipirona & $6,34 \% \pm 0,110$ & Bibiana et al., 2019; Dutra et al., 2019; Santos et al., 2012; Tavares et al., 2018. \\
\hline Atenolol X Ibuprofeno & $12,1 \% \pm 0,153$ & Bibiana et al., 2019; Tavares et al., 2018. \\
\hline Captopril X Dipirona & $19,63 \% \pm 0,305$ & Bibiana et al., 2019; Santos et al., 2012; Tavares et al., 2018. \\
\hline Diclofenaco X Enalapril & $1,12 \% \pm 0,009$ & Bibiana et al., 2019; Santos et al., 2012. \\
\hline Diclofenaco X Losartana & $7,30 \% \pm 0,092$ & Bibiana et al., 2019; Santos et al., 2012. \\
\hline Dipirona X Enalapril & $14,3 \% \pm 0,196$ & Bibiana et al., 2019; Dutra et al., 2019; Tavares et al., 2018. \\
\hline Dipirona X Losartana & $2,18 \% \pm 0,014$ & Bibiana et al., 2019; Dutra et al., 2019; Santos et al., 2012. \\
\hline Dipirona X Propranolol & $18,2 \% \pm 0,206$ & Bibiana et al., 2019; Tavares et al., 2018. \\
\hline Enalapril X Ibuprofeno & $5,21 \% \pm 0,054$ & Amaral \& Perassolo, 2012; Bibiana et al., 2019. \\
\hline Ibuprofeno X Propranolol & $12,1 \% \pm 0,192$ & Amaral \& Perassolo, 2012; Bibiana et al., 2019; Tavares et al., 2018. \\
\hline
\end{tabular}

Siglas: AAS, ácido acetilsalicílico; DP, Desvio Padrão. Fonte: Dados da Pesquisa (2020).

Podem reduzir o efeito do anti-hipertensivo interações entre AAS com captopril ou enalapril. Podem elevar a PA, interações entre AAS e atenolol ou propranolol, atenolol e dipirona, atenolol e ibuprofeno, captopril e dipirona, dipirona e enalapril, ibuprofeno e propranolol. Podem provocar uma disfunção renal e ou o aumento da PA, as combinações entre: diclofenaco e enalapril, diclofenaco e losartana, enalapril e ibuprofeno, dipirona e losartana, dipirona e propranolol. As médias proporcionais de IM entre anti-hipertensivos e outras classes farmacológicas encontradas estão representadas na Tabela 5 . 
Tabela 5: Interação medicamentosa considerada nível moderado D: anti-hipertensivos x outras classes.

\begin{tabular}{lcc}
\hline Interação medicamentosa & Média e DP & Referências \\
\hline Fluconazol x Losartana & $0,95 \% \pm 0,82$ & Bibiana et al., 2019; Dutra et al., 2019. \\
Fluoxetina x Nifedipino & $0,22 \% \pm 0,21 \quad$ Bibiana et al., 2019; Santos et al., 2012. \\
$\begin{array}{l}\text { Levotiroxina x Sinvastatina } \\
\text { Omeprazol x Propranolol }\end{array}$ & $26,9 \% \pm 32,7$ & Bibiana et al., 2019; Tavares et al., 2018. \\
Cálcio + Colecalciferol (Vit D)x & $3,05 \% \pm 3,72$ & Bibiana et al., 2019; Dutra et al., 2019. \\
$\quad$ Hidroclorotiazida & $0,53 \% \pm 0,23$ & Amaral \& Perassolo, 2012; Bibiana et al., 2019. \\
\hline
\end{tabular}

Sigla: DP, Desvio Padrão. Fonte: Dados da Pesquisa (2020).

A interação entre fluconazol e losartana pode diminuir a conversão de losartana em seu metabólito ativo. Já fluoxetina e nifedipino pode causar em exposição ao aumento da Nifedipina. Entre levotiroxina e sinvastatina pode reduzir a eficácia da levotiroxina. E omeprazol e propranolol pode aumentar a exposição ao propranolol. Cálcio, colecalciferol (Vitamina D) e hidroclorotiazida podem elevar o risco de hipercalcemia.

As IM com anti-hipertensivos, consideradas nível grave, também foram separadas por grupos, a saber: IM entre antihipertensivos e anti-inflamatórios não esteroides (AINES) (Tabela 6); IM entre anti-hipertensivos e outras classes farmacológicas (Tabela 7).

As médias proporcionais de IM entre anti-hipertensivos e anti-inflamatórios não esteroides (AINES) encontradas estão representadas na Tabela 6.

Tabela 6: Interação medicamentosa considerada nível grave A: anti-hipertensivos x AINES.

\begin{tabular}{|c|c|c|}
\hline Interação medicamentosa & Média e DP & Referências \\
\hline AAS x Furosemida & $4,43 \% \pm 0,115$ & Bibiana et al., 2019; De Lima \& Godoy, 2017; Santos et al., 2012. \\
\hline AAS x Hidroclorotiazida & $3,36 \% \pm 0,072$ & Santos et al., 2012; Tavares et al. (2018), Bibiana et al. (2019) \\
\hline Diclofenaco x Furosemida & $1,7 \% \pm 0,0008$ & Bibiana et al., 2019; Santos et al., 2012. \\
\hline Dipirona $\mathrm{x}$ Espironolactona & $1,96 \% \pm 0,009$ & Bibiana et al., 2019; Santos et al., 2012; Santos et al., 2019. \\
\hline Dipirona X Furosemida & $2,26 \% \pm 0,012$ & Bibiana et al., 2019; Santos et al., 2012. \\
\hline Dipirona x Hidroclorotiazida & $2,04 \% \pm 0,024$ & Bibiana et al., 2019; Dutra et al., 2019; Santos et al., 2012. \\
\hline Ibuprofeno x Furosemida & $1,79 \% \pm 0,021$ & Bibiana et al., 2019; Tavares et al., 2013. \\
\hline
\end{tabular}

Siglas: AAS, ácido acetilsalicílico; DP, Desvio Padrão. Fonte: Dados da Pesquisa (2020).

Pode acontecer a diminuição da eficácia do diurético e possível nefrotoxicidade quando há interações entre AAS e furosemida, AAS e hidroclorotiazida, diclofenaco e furosemida, dipirona e espironolactona, dipirona e furosemida, dipirona e hidroclorotiazida, furosemida e ibuprofeno.

As médias proporcionais de IM entre anti-hipertensivos e outras classes farmacológicas encontradas estão representadas na Tabela 7. 
Tabela 7: Interação medicamentosa considerada nível grave B: anti-hipertensivos x outras classes farmacológicas.

\begin{tabular}{|c|c|c|}
\hline Interação medicamentosa & Média e DP & Referências \\
\hline Amiodarona X Ciprofloxacino & $0,57 \% \pm 0,003$ & Bibiana et al., 2019; Dutra et al., 2019. \\
\hline Amiodarona X Sinvastatina & $2,08 \% \pm 0,023$ & $\begin{array}{l}\text { Bibiana et al., 2019; Pinto et al., 2014; Santos et al., 2012; Tavares et al., } \\
2018 .\end{array}$ \\
\hline Amiodarona X Tramadol & $0,3 \% \pm 0,0005$ & Bibiana et al., 2019; Dutra et al., 2019; \\
\hline Anlodipino X Sinvastatina & $17,1 \% \pm 0,122$ & $\begin{array}{l}\text { Bibiana et al., 2019; Dutra et al., 2019; Leão et al., 2020; Neiva et al., } \\
\text { 2019; Petri et al., 2020; Pinto et al., 2014; Rocha et al., 2017; Santos et al., } \\
\text { 2019; Spanevello, 2018; Tavares et al., 2018. }\end{array}$ \\
\hline Digoxina X Espironolactona & $3,57 \% \pm 0,020$ & Bibiana et al., 2019; Spanevello, 2018; Tavares et al., 2018. \\
\hline Digoxina X Hidroclorotiazida & $9,12 \% \pm 0,133$ & Amaral \& Perassolo, 2012; Bibiana et al., 2019; Tavares et al., 2013. \\
\hline Enalapril X Espironolactona & $3,66 \% \pm 0,019$ & Bibiana et al., 2019; Petri et al., 2020; Pinto et al., 2014. \\
\hline
\end{tabular}

Sigla: DP, Desvio Padrão. Fonte: Dados da Pesquisa (2020).

Combinação de sinvastatina com amiodarona ou anlodipino pode aumentar exposição à sinvastatina e provoca um risco maior de miopatia ou rabdomiólise. A associação de amiodarona e tramadol pode aumentar exposição do tramadol e gerar risco maior de depressão respiratória. Já em relação ao uso conjugado de digoxina e espironolactona, pode aumentar a exposição à digoxina. E a combinação de digoxina e hidroclorotiazida pode gerar toxicidade digitálica (náuseas, vômitos, arritmias). Por fim, a combinação entre enalapril e espironolactona pode resultar em hipercalemia.

As interações medicamentosas entre anti-hipertensivos que foram frequentes nos últimos 20 anos estão representadas nas figuras abaixo separadas por grupos: IM entre anti-hipertensivos e outros fármacos que atuam no sistema cardiovascular, podendo ser outro fármaco anti-hipertensivo (Figura 3); IM entre anti-hipertensivos e hipoglicemiantes (Figura 4); IM entre anti-hipertensivos e anti-inflamatórios não esteroides (AINES) (Figura 5); IM entre anti-hipertensivos e outras classes farmacológicas (Figura 6).

As IM mais frequentes entre diferentes classes de anti-hipertensivos, incluindo alguns diuréticos que também são classificados na classe terapêutica anti-hipertensivos, estão representadas na Figura 3.

As médias e DP sobre a frequência de IM entre entre anti-hipertensivos e outros fármacos que atuam no sistema cardiovascular representadas na Figura 3 foram 5,79\% ( $\pm 7,08 \%)$ entre captopril e furosemida, 8,05\% ( $\pm 5,29 \%)$ entre captopril e hidroclorotiazida, $4,10 \%( \pm 2,06 \%)$ entre captopril e propranolol, $19,44 \%( \pm 24,55 \%)$ entre enalapril e digoxina, $15,39 \%$ $( \pm 12,42 \%)$ entre enalapril e furosemida, $6,70 \%( \pm 7,06 \%)$ entre enalapril e hidroclorotiazida, e por fim, $3,85 \%( \pm 2,08 \%)$ entre hidroclorotiazida e propranolol. 
Figura 3: Interações medicamentosas mais frequentes: anti-hipertensivos $x$ outros fármacos que atuam no sistema cardiovascular.

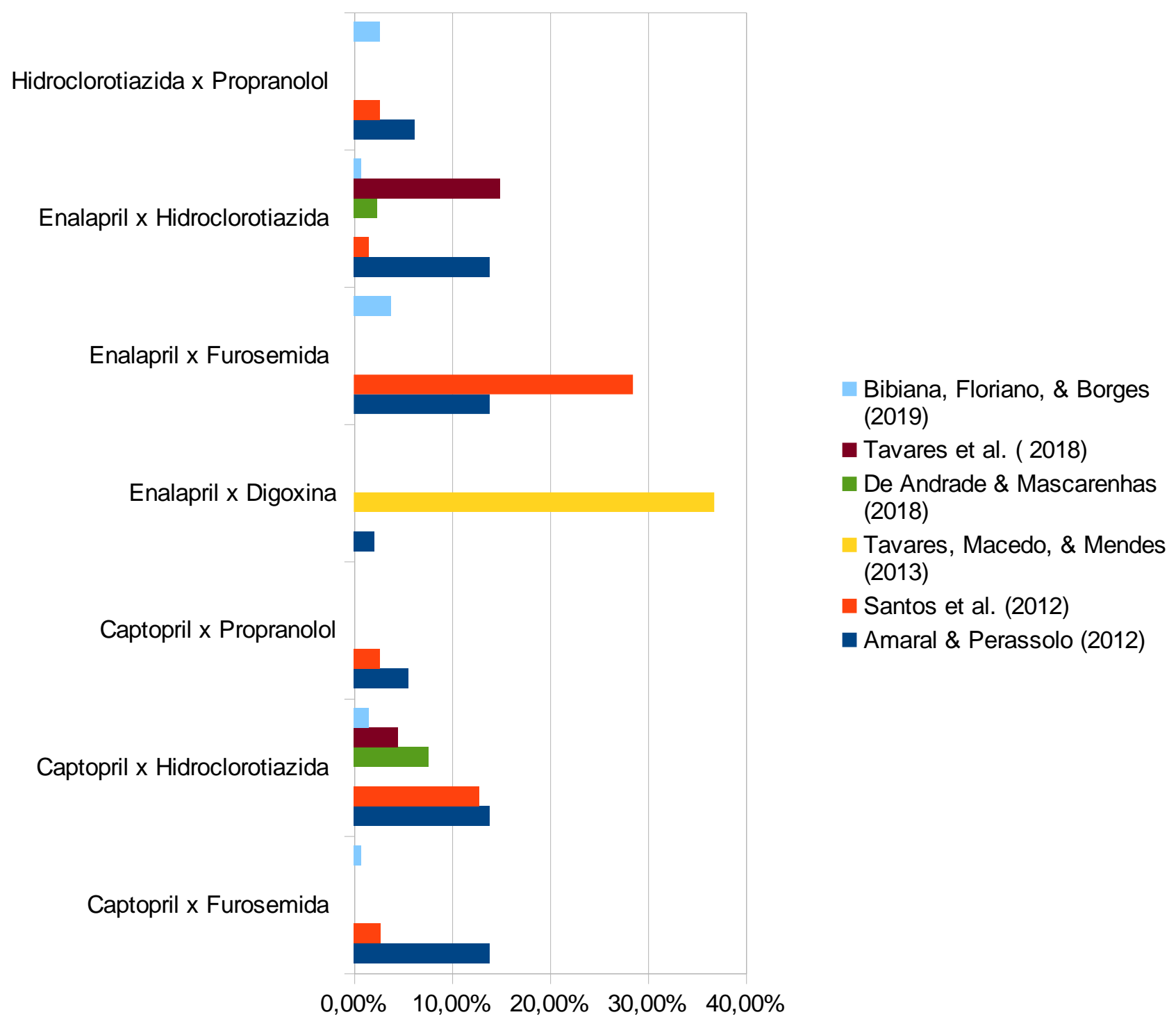

Fonte: Dados da Pesquisa (2020).

As IM mais frequentes entre anti-hipertensivos e hipoglicemiantes estão representadas na Figura 4, sendo que as médias e DP dessas IM foram 5,80\% ( $\pm 0,04 \%)$ entre captopril e glibenclamida, 6,82\% $( \pm 0,02 \%)$ entre enalapril e glibenclamida, 8,21\% $( \pm 0,05 \%)$ entre enalapril e metformina, 8,43\% $( \pm 0,07 \%)$ entre enalapril e insulina $\mathrm{NPH}, 7,33 \%$ $( \pm 0,04 \%)$ entre glibenclamida e hidroclorotiazida, 7,68\% $( \pm 0,03 \%)$ entre metformina e hidroclorotiazida.

As interações medicamentosas mais frequentes entre anti-hipertensivos e anti-inflamatórios não esteroides (AINES) estão representadas na Figura 5, sendo que as médias e DP dessas IM foram 10,05\% ( $\pm 0,098 \%)$ entre anlodipino e AAS (ácido acetilsalicílico), $16,42 \%( \pm 0,21 \%)$ entre atenolol e AAS, 7,15\% ( $\pm 0,08 \%)$ entre captopril e AAS, $13,84 \%$ entre enalapril e AAS relatada em um único estudo (Bibiana et al., 2019), 7,30\% ( $\pm 0,09 \%)$ entre losartana e diclofenaco, 6,34\% $( \pm 0,11 \%)$ entre atenolol e dipirona, $19,63 \%( \pm 0,31 \%)$ entre captopril e dipirona, $14,32 \%( \pm 0,20 \%)$ entre enalapril e dipirona, $6,05 \%( \pm 0,05 \%)$ entre furosemida e dipirona, $18,16 \%( \pm 0,21 \%)$ entre propranolol e dipirona, $12,06 \%( \pm 0,15 \%)$ entre atenolol e ibuprofeno, $5,21 \%( \pm 0,05 \%)$ entre enalapril e ibuprofeno e, finalmente, $12,13 \%( \pm 0,19 \%)$ entre propranolol e ibuprofeno. 
Figura 4: Interações medicamentosas mais frequentes: anti-hipertensivos x Hipoglicemiantes.

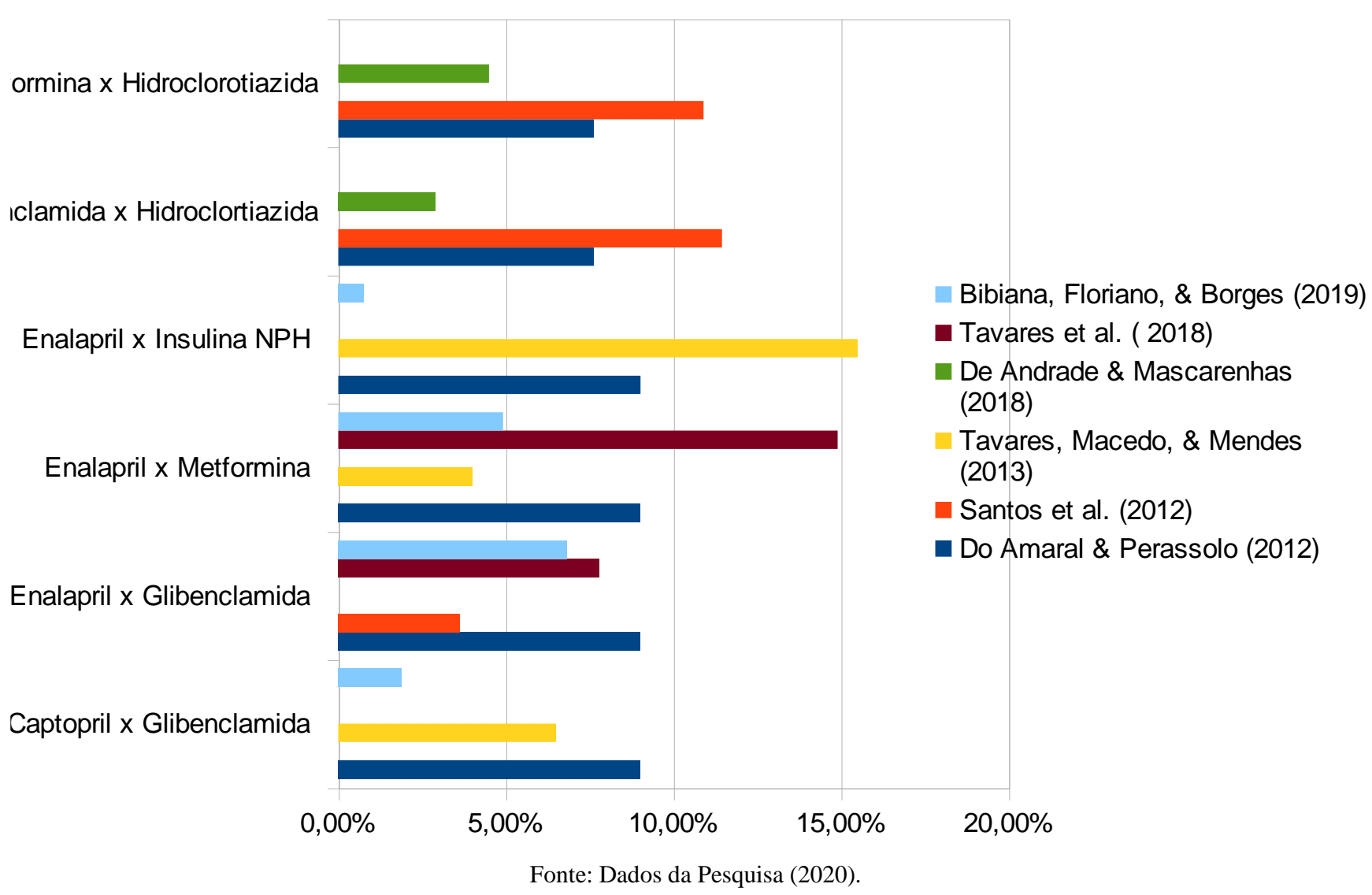

Figura 5: Interações medicamentosas mais frequentes: anti-hipertensivos x AINES.

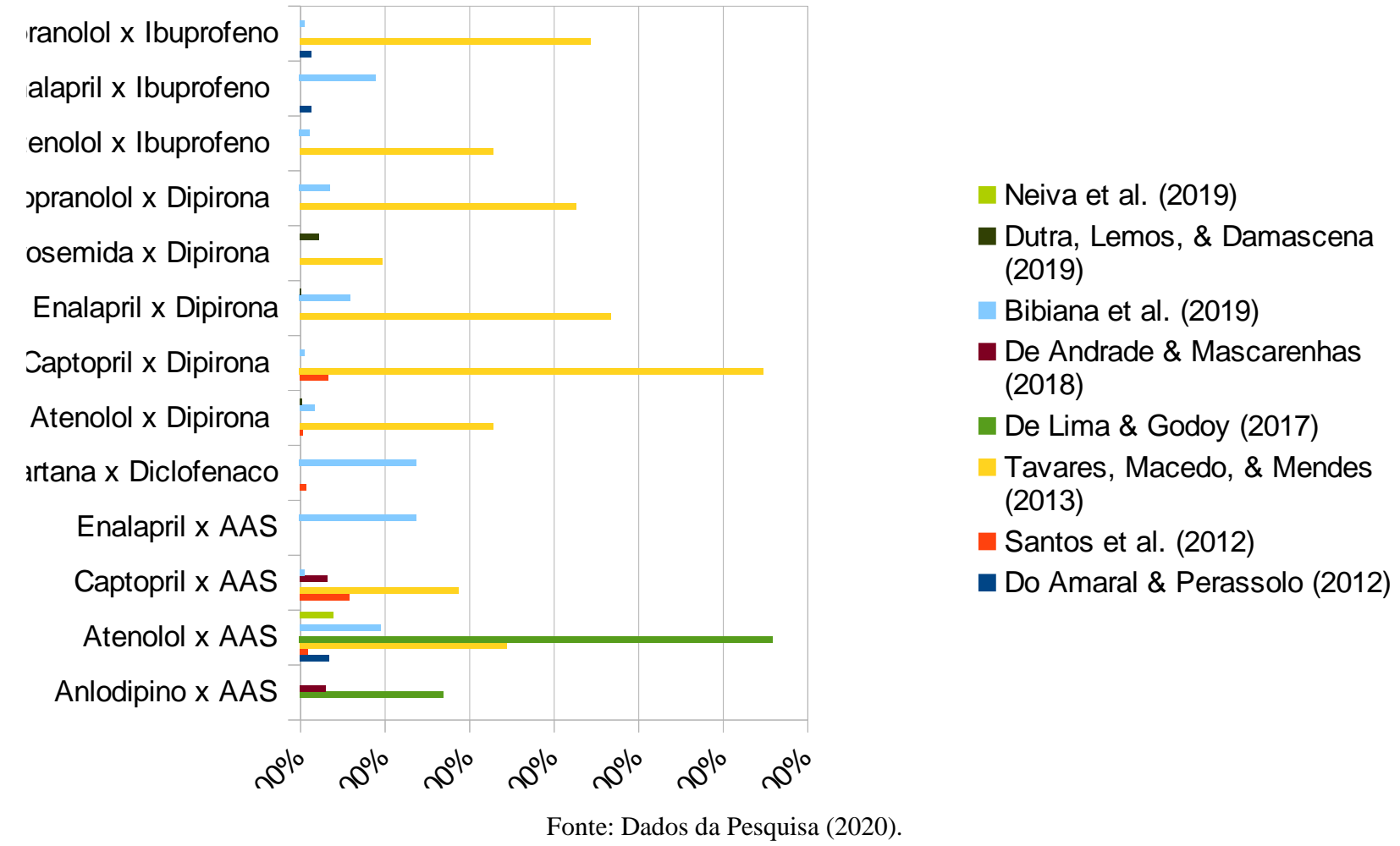


As IM mais frequentes entre anti-hipertensivos e outras classes farmacológicas estão representadas na Figura 6. As respectivas médias e desvios padrão dessas IM foram $17,10 \%( \pm 0,12 \%)$ entre anlodipino e sinvastatina, $9,12 \%( \pm 0,13 \%)$ entre hidroclorotiazida e digoxina, $13,29 \%$ ( $\pm 0,16 \%)$ entre captopril e digoxina, $7,10 \%( \pm 0,12 \%)$ entre furosemida e digoxina.

Figura 6: Interações medicamentosas mais frequentes: anti-hipertensivos x outras classes farmacológicas.

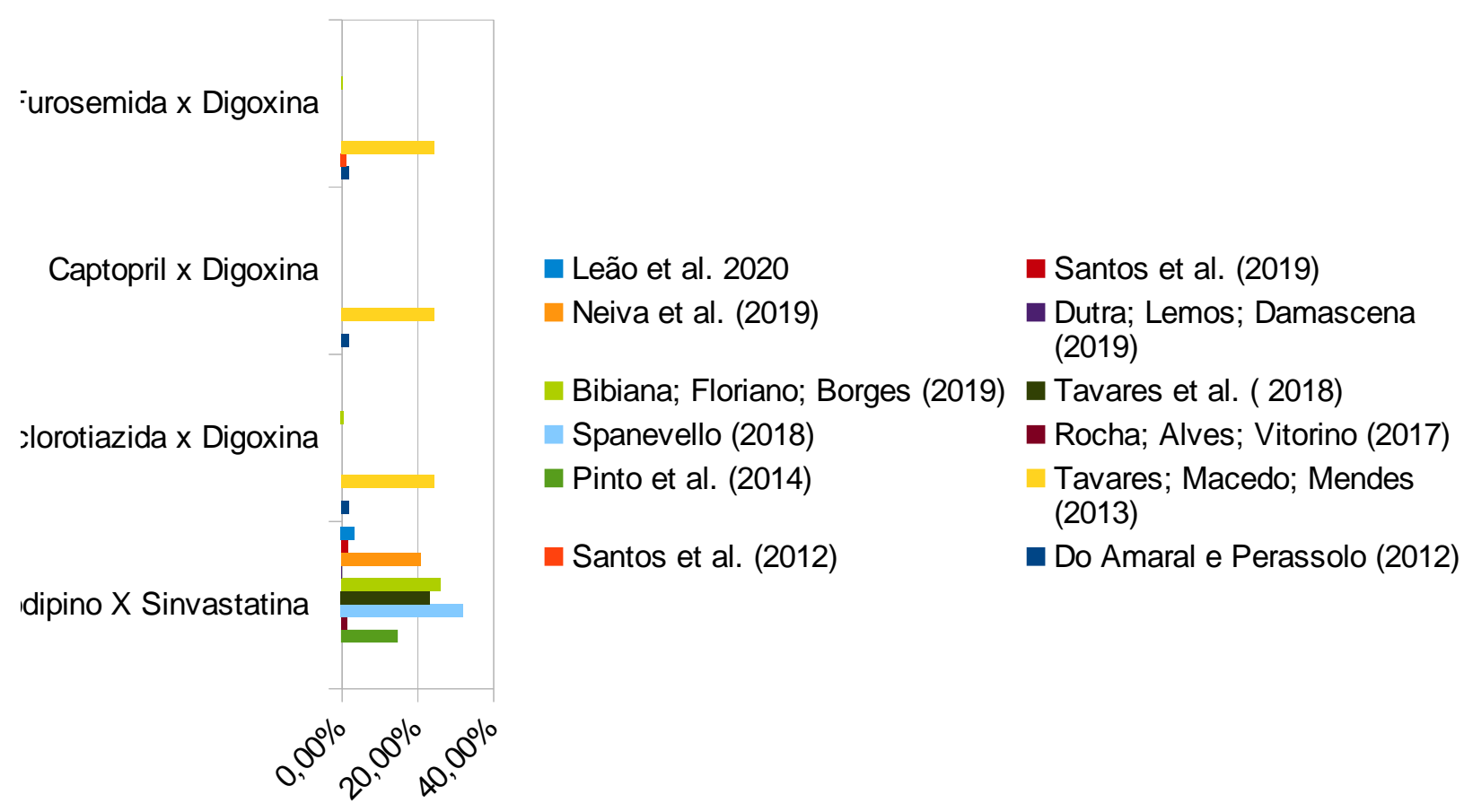

Fonte: Dados da Pesquisa (2020).

\section{Discussão}

Segundo Zanetti et al. (2017) os fármacos que mais estão ligados as possíveis interações são os que atuam no sistema cardiovascular, os anti-inflamatórios não esteroides (AINE), os antidiabéticos e os antiácidos. Já no estudo de Petri et al. (2020) as classes farmacológicas que mais destacam em relação a frequência de IM foram: anti-hipertensivos, anticoagulantes, antibióticos e analgésicos, sendo que os medicamentos mais frequentes nas IM foram: propranolol com dipirona, varfarina com heparina, propranolol com furosemida e dipirona com captopril.

Medicamentos como AINE, betabloqueadores, inibidores da enzima conversora de angiotensina (IECA), diuréticos, digoxina, antilipêmicos, depressores do sistema nervoso central são potencialmente interativos. Os AINE podem até ser indicados para pacientes que utilizam anti-hipertensivos, desde que seja a curto prazo e que não tenham doença cardíaca grave. Mas deve ser evitado por pacientes idosos ou aqueles que tenha deficiência renal ou que fazem uso da digoxina. Além disso, a associação dos AINE e diuréticos tiazídicos, assim como, a combinação de AINE e IECA, podem gerar alteração da função renal, desequilíbrio eletrolítico e comprometer a eficácia da farmacoterapêutica anti-hipertensiva (Bertollo et al., 2014; Secoli, 2010).

A classe farmacológica dos AINE está envolvida em várias interações com anti-hipertensivos, inibindo os efeitos dos anti-hipertensivos dos diuréticos tiazídicos e de alça, os antagonistas de receptores alfa e dos receptores beta-adrenérgicos, assim como os agentes que bloqueiam o sistema renina-angiotensina-aldosterona. De acordo com Bertollo et al. (2014) o diclofenaco de sódio e ibuprofeno reduzem o efeito dos diuréticos e casos como esses, deve-se considerar a modificação de 
terapia. E pesquisas clínicas estudadas por Bibiana et al. (2019) relatam que os AINE, tanto os seletivos como não seletivos, podem provocar ou agravar a hipertensão arterial já presente.

Observou-se que pacientes diabéticos, tiveram IM recorrentes entre os antagonistas dos receptores de angiotensina II e IECA com os diuréticos, pois os fármacos dessas classes farmacológicas possuem mecanismos de ação semelhantes, em razão da farmacodinâmica dos grupos envolvidos. Indivíduos com diabetes e insuficiência cardíaca, o risco de hipotensão por causa da junção de IECA ou antagonistas dos receptores da angiotensina II com diuréticos se mostra muito importante clinicamente. Essa hipotensão é resultante da depleção de sódio pelo inibidor da enzima conversora de angiotensina, intensificada pela ação adicional do diurético. Apesar dessa combinação aumentar a natriurese, os IECA também podem diminuir a filtração glomerular, a diurese e as respostas natriuréticas aos diuréticos (Santos et al., 2019).

Amaral e Perassolo (2012) relataram duas interações entre antagonista de canal de cálcio e diurético. Esta interação colabora na mortalidade cardiovascular em mulheres que estão na fase pós-menopausa quando comparada ao uso de diurético associado a betabloqueador. Além disso, observaram possíveis IM entre anti-hipertensivos e antidiabéticos em participantes do grupo HIPERDIA. Notaram que o consumo de glibenclamida ou metformina coadministrados com hidroclorotiazida pode elevar a glicemia por reduzir o efeito desses hipoglicemiantes.

E na pesquisa de Andrade e Souza (2018), notificaram 4,5\% de interações envolvendo os medicamentos metformina, hipoglicemiante hidroclorotiazida, anti-hipertensivo, as quais foram consideradas como IM moderada. Já em Leão et al. (2020), uma das IM mais frequentes foi entre hidroclorotiazida e glibenclamida $(11,45 \%)$ e hidroclorotiazida com metformina $(10,89 \%)$.

A digoxina é um fármaco digitálico classificado de alto risco devido ao baixo índice terapêutico, e deve ser avaliado o risco-benefício cuidadosamente, pois ele esteve presente em 1,18\% do total de prescrições. Além disso, está frequentemente ligado a diversas IM, sobretudo naquelas que envolvem a farmacocinética e podem aumentar as concentrações séricas da digoxina, como: quinidina, verapamil, amiodarona, espironolactona, triantereno, eritromicina. Sabe-se também, que a hipopotassemia, hipomagnesemia, hipercalcemia, hipóxia, acidose predispõem à intoxicação digitálica (Dos Santos et al., 2012).

A interação de gravidade leve entre aspirina e atenolol $(3,95 \%)$ foi a terceira mais predominante. A ação de um betabloqueador na diminuição da pressão arterial pode ser reduzida por salicilatos; consequentemente, é necessário o monitoramento do paciente que faz a coadministração desses dois medicamentos. E nesse caso, o médico pode reduzir a dose do salicilato ou alterar a terapêutica anti-hipertensiva. Foi verificado também, interações graves e a segunda mais frequente ocorreu entre o atenolol e clonidina que se destacaram em 10,33\% das prescrições. A interação entre um betabloqueador e o fármaco clonidina pode gerar hipertensão rebote no hipertenso, sendo indicado ajuste na terapia medicamentosa para evitar esse tipo de reação (Neiva et al., 2019).

Pinto et al. (2014) obtiveram 42,5\% de interação entre AAS e enalapril, considerada de nível moderada. E em relação as interações da glibenclamida com AAS e enalapril, identificadas neste estudo, têm capacidade de provocar hipoglicemia excessiva. Assim, o risco dessa interação demonstra a necessidade de monitorar os pacientes, ou quando possível, substituir a sulfonilureia por outra classe mais apropriada para idosos, de acordo com o Critério de Beers da American Geriatric Society (2012), pois possui alto risco de produzir hipoglicemia grave prolongada em idosos.

Em relação as interações classificadas como indesejáveis, muito graves, a associação de alopurinol e enalapril podem causar graves reações de hipersensibilidade, como a síndrome de Stevens-Johnson, artralgia, febre e anafilaxia. (Gonzaga et al., 2009). Em Spanevello (2019) essa combinação representou 6,45\% das interações medicamentosas. Nesse caso, é fundamental que o farmacêutico informe a probabilidade de reações adversas relacionadas a essa interação e as principais manifestações clínicas que ela pode causar (Amaral \& Perassolo, 2012). 
Segundo Spanevello (2018), um quinto das IM observadas foram consideradas graves, demonstrando o quanto é necessário a intervenção da equipe de saúde para evitar prejuízos à saúde dos pacientes. Das IM consideradas graves, 32,26\% foram entre anlodipino e sinvastatina que podem gerar rabdomiólise. Esse fenômeno está ligado a liberação de substancias intracelulares, possivelmente tóxicas na circulação, exemplo, a mioglobina, que é nefrotóxico, o que pode causar ou piorar danos renais. E Leão et al. (2020) também verificaram a mesma combinação entre anlodipino e sinvastatina cuja porcentagem foi de $3,63 \%$.

Em outro estudo, também foi reportado a interação grave entre, anlodipino e sinvastatina, e que foi a mais frequente observada 15\% das receitas médicas (Pinto et al., 2014). Em Neiva et al. (2019) foi muito frequente a junção entre anlodipino e sinvastatina, 20,97\% das prescrições. Essa combinação eleva o risco de casos de miopatias e rabdomiólise, pois o anlodipino aumenta os níveis séricos das estatinas por meio de alterações farmacocinéticas. Portanto, não se pode ultrapassar de 20 mg por dia de sinvastatina quando coadministrado com anlodipino.

Na pesquisa de Bibiana et al. (2019), também foi observada alta porcentagem da combinação entre anlodipino e sinvastatina, 26,28\%. Devido a frequente coadministração de fármacos nas doenças associadas a HAS, como hiperlipidemia, aumento do colesterol, doenças cardiovasculares, indica-se a administração em espaço de horas entre o anlodipino e a sinvastatina.

\section{Considerações Finais}

De modo geral, uma das classes farmacológicas mais envolvidas em interações medicamentosas é a classe terapêutica anti-hipertensivos, que se subdivide em diversas classes farmacológicas com o mesmo uso terapêutico. Assim, fármacos antihipertensivos de diferentes classes podem interagir entre si e com outras classes de medicamentos. Em nossa revisão sistemática, as classes farmacológicas mais frequentes causadoras de IM com os anti-hipertensivos foram: hipoglicemiantes, AINES, diuréticos e entre dois tipos de anti-hipertensivos.

Da classe farmacológica dos AINES, o AAS foi o fármaco que mais se destacou em IM relacionada a variedade de combinações com anti-hipertensivos, os quais podemos citar de acordo com porcentagem em ordem decrescente furosemida, hidroclorotiazida e captopril. A dipirona, foi o segundo da mesma categoria que interagiu com mais de um anti-hipertensivo, entre eles, captopril, enalapril e propranolol.

Já a classe dos hipoglicemiantes, os que mais se destacaram foram a insulina, metformina e glibenclamida. Em que a insulina interagiu mais com enalapril e furosemida; a metformina interagiu mais com enalapril, hidroclorotiazida e captopril; a glibenclamida interagiu mais com enalapril, captopril e hidroclorotiazida.

Em se tratando de IM grave entre anti-hipertensivos e fármacos de diferentes classes farmacológicas, a digoxina foi o fármaco que mais se destacou, seguido pela sinvastatina.

Assim, dos anti-hipertensivos mais frequentes em interações, se destacam: captopril, enalapril e hidroclorotiazida. Já os fármacos que mais se destacaram, causando algum prejuízo ao efeito farmacológico dos anti-hipertensivos foram: AAS, dipirona e digoxina. E a sinvastatina, apesar de não interferir no efeito farmacológico quando associado ao anti-hipertensivo (anlodipino), pode gerar danos à saúde. Desse modo, algumas das IM apresentadas podem colocar em risco a saúde do paciente, provando a importância deste tema e o indispensável monitoramento terapêutico, no sentido de prevenir e reduzir as consequências das potenciais IM.

É essencial o conhecimento sobre esse assunto, pois possibilita a execução de estratégias e protocolos que ajudem a equipe de saúde a detectar potenciais interações e empregar medidas de prevenção e monitorização de pacientes com grandes chances de sofrer IM. Além disso, o farmacêutico clínico tem grande relevância nas equipes de saúde, devido a sua capacidade de ajudar no rastreamento de eventos adversos a medicamentos e na otimização da farmacoterapia. 
Contudo, essas intervenções farmacêuticas podem propiciar a redução de erros relacionados aos medicamentos prescritos, favorecendo para o sucesso do tratamento, eficácia terapêutica e principalmente para a segurança do paciente. É indispensável que mais pesquisas sejam desenvolvidas, relacionadas a esse tema e demais classes terapêuticas, para que os profissionais farmacêuticos permaneçam atualizados e tenham reconhecimento do seu valor diante da equipe multidisciplinar contribuindo no tratamento e melhora do paciente.

\section{Agradecimentos}

Agradecemos a Marilza Aparecida Machado Borges pelo auxílio a correção gramatical.

\section{Referências}

Amaral, D. M. D. \& Perassolo, M. S. (2012) Possíveis interações medicamentosas entre os antihipertensivos e antidiabéticos em participantes do Grupo HIPERDIA de Parobé, RS (Uma análise teórica). Revista de Ciências Farmacêuticas Básica e Aplicada, 33 (1), 99-105.

American Geriatrics Society (2012). Beers Criteria Up-date Expert Panel. American Geriatrics Society updated Beers Criteria for potentially inappropriate medication use in older adults. J Am Geriatr Soc., 60, 616-31.

Andrade, K. V. F. \& Souza, A. M. (2018) Prevalência de interações medicamentosas potenciais em indivíduos hipertensos acompanhados na estratégia de saúde da família. Journal of Health \& Biological Sciences, 6 (4), 405-411.

Bertollo, A. L., Demartini, C., \& Piato, A. L. (2014). Interações medicamentosas na clínica odontológica. Revista Brasileira de Odontologia, 70 (2), 120.

Bibiana, B. G., Floriano, S. R., \& Borges, M. S. (2019). Avaliação das interações medicamentosas em prontuários de pacientes de uma unidade básica de saúde. Journal of applied pharmaceutical sciences, 6, 9-27.

Brasil (2012). Ministério da Saúde. Secretaria de Ciência, Tecnologia e Insumos Estratégicos. Uso racional de medicamentos: temas selecionados - Série A. Normas e Manuais Técnicos. Brasília: Ministério da Saúde, 1-158.

Brasil (2013). Secretaria de Atenção à Saúde. Departamento de Atenção Básica. Estratégia para o cuidado da pessoa com doença crônica: hipertensão arterial sistêmica. Cadernos de Atenção Básica. Brasília: Ministério da Saúde, 1 (37), 1-130.

Brasil (2008). Ministério da Saúde. Secretaria de Ciência, Tecnologia e Insumos Estratégicos. Departamento de Assistência Farmacêutica e Insumos Estratégicos. Formulário terapêutico nacional 2008. Ministério da Saúde, Secretaria de Ciência, Tecnologia e Insumos Estratégicos, Departamento de Assistência Farmacêutica e Insumos. Estratégicos. Brasília: Ministério da Saúde. (Série B. Textos Básicos de Saúde). p. 26. http://bvsms.saude.gov. br/bvs/publicações/formulário_terapeutico_nacional_2008.pdf

Campana, É. M. G., Lemos, C. C., Magalhães, M. E. C., Brandão, A. A., \& Brandão, A. P. (2009). Interações e associações medicamentosas no tratamento da hipertensão Bloqueadores alfa-adrenérgicos e vasodilatadores diretos. Rev. Bras. Hipertens., 16 (4), 231-236.

Cardoso, C. M. Z., Silva, C. P., Yamagami, K., Lopes, R. P., Santos, F., Bonassi, I., Jesuíno, I., Geres, F., ... Inowe, C. (2009). Elaboração de uma cartilha direcionada aos profissionais da Área da Saúde, Contendo Informações sobre Interações Medicamentosas envolvendo Fitoterápicos e Alopáticos. Revista Fitos, 4 (1), 56-69.

Carreira C. F. S., Barrêto V. F. T., Moura, A. P. G., Silva, P. R. J., Teixeira, N. A. M., Canavieiras, S. A. (2008). Interações medicamentosas: um relato de caso sobre a avaliação e intervenção farmacêutica. In: XI Encontro De Iniciação À Docência. Anais ENID. João Pessoa: UFPB-PRG, 2008. http://www.prac.ufpb.br/anais/xenex_xienid/xi_enid/monitoriapet/ANAISArea6/6CCSDCFPET02.pdf

Carvalho, F. A., Biella, C. A., \& Graciani, F. S. (2017). Riscos da interação medicamentosa em pacientes hipertensos: um estudo em grupo específico de pacientes que fazem uso de anti-hipertensivos. Journal of the Health Sciences Institute, 35 (3), 215-8.

De Lima, T. A. M. \& De Godoy, M. F. (2017) Interações medicamentosas em prescrições para idosos hospitalizados com Síndrome Coronariana Aguda. Revista Eletrônica de Enfermagem, 19.

Dos Santos, J. C., Faria Jr., M., \& Restini, C. B. A. (2012). Potenciais interações medicamentosas identificadas em prescrições a pacientes hipertensos. Rev Bras Clin Med, 10 (4), 308-17.

Dutra, A. P. R., Lemos, L. M. A., \& Damascena, R. S. (2019). Avaliação do Perfil das Interações Medicamentosas e os Fatores Associados em Prescrições Médicas de Pacientes Internados em Unidade de Terapia Intensiva. ID on line Revista de Psicologia, 13 (43), 543-558.

Eschenhagen, T. (2019) Tratamento da hipertensão. In L. L. Brunton, R. H. Dandan, \& B. C. Knollmann. As bases farmacológicas da terapêutica de Goodman \& Gilman. Porto Alegre: AMGH, 625-649.

Faria, A. L. G., Fais, F. L. B., Ribeiro, J. M., Marialva, R. L. H., Costa, V. D., \& Matsutani, G. C. (2019). Avaliação das interações medicamentosas e possíveis efeitos colaterais em pacientes idosos da clínica cardiovascular. Diálogos Interdisciplinares, 8 (10), 21-28.

Fava, S. M. C. L., Da Silva, P. C. dos S., Gonçalves, I. W. P., Gomes, D. M., Machado, J. P., \& Veiga, E. V. (2017) Classes de anti-hipertensivos e sua combinação entre pessoas com hipertensão arterial sistêmica no sistema público. Enfermería Global, 16 (45) 20-50. 
Giles, T. D., Materson, B. J., Cohn, J. N., \& Kostis, J. B. (2009). Definition and classification of hypertension: an update. The Journal of Clinical Hypertension, 11 (11), 611-614.

Gomes, A. M. P., Bezerra, K. G. D., \& Oliveira, F. S. (2019). Avaliação de potenciais interações medicamentosas em pacientes da unidade de terapia intensiva de um hospital universitário. Revista de Ciências Médicas e Biológicas, 18 (2), 183-189.

Gomes, A. M. P., Dias Bezerra, K. G., \& Oliveira, F. de S. (2019). Avaliação de potenciais interações medicamentosas em pacientes da unidade de terapia intensiva de um hospital universitário. Revista De Ciências Médicas E Biológicas, 18(2), 183-189. https://doi.org/10.9771/cmbio.v18i2.29253

Gontijo, M. D. F., Ribeiro, A. Q., Klein, C. H., Rozenfeld, S., \& Acurcio, F. D. A. (2012). Uso de anti-hipertensivos e antidiabéticos por idosos: inquérito em Belo Horizonte, Minas Gerais, Brasil. Cadernos de Saúde Pública, 28, 1337-1346.

Gonzaga, C. C., Passarelli J. R. O., \& Amodeo, C. Interações medicamentosas: inibidores da enzima conversora da angiotensina, bloqueadores dos receptores da angiotensina II, inibidores diretos da renina. Rev Bras Hipertens, 16 (4), 221-225.

Hoffman, B. B. \& Michel, T. (2012). Tratamento da isquemia miocárdica e da hipertensão. In: Goodman, L.S.; Gilman, A.; Brunton, L.; Knollman, B.; Chabner, B. As Bases Farmacológicas da Terapêutica de Goodman e Gilman. 12ed. Porto Alegre: AMGH, 745-788.

Ioris, L. M. D. \& Bacchi, A. D. (2019) Interações medicamentosas de interesse em odontologia. Revista da Faculdade de Odontologia-UPF, 24 (1), 148-154.

Jacomini, L. C. L. \& Silva, N. A. (2011). Interações medicamentosas: uma contribuição para o uso racional de imunossupressores sintéticos e biológicos. Rev. Brasileira de Reumatologia, 51 (2), 168-174.

Lattuca, B., Khoueiry, Z., Malclès, G., Davy, J. M., \& Leclercq, F. (2013). Drug interactions between non-steroidal anti-inflammatory drugs and cardiovascular treatments (except anti-agregant therapy). Anti-Inflammatory \& Anti-Allergy Agents in Medicinal Chemistry, 12 (1), $36-46$.

Leão, I. N., Fernandes, B. D., De Oliveira, B. G., Almeida, P. H. R. F., Da Silveira Lemos, G., Junior, G. L. V ; Mascarenhas, C. H. M. (2020). Prevalência das interações medicamentosas potenciais e suas possíveis consequências clínicas em indivíduos hipertensos atendidos na atenção primária. Revista de Atenção à Saúde, 18 (63), 2020.

Luz, V., Marques, M. S., \& De Jesus, N. N. (2018). Riscos de Interações Medicamentosas Presentes nos Receituários de Pacientes Hipertensos e Diabéticos: Uma Revisão Bibliográfica. ID on line Revista de Psicologia, 12 (40), 793-806.

Matos, V. T., Vasconcelos, E. F., Amaral, M. S. D., \& Toffoli-Kadri, M. C. (2009). Avaliação das interações medicamentosas em prescrições hospitalares de pacientes sob uso de anti-hipertensivos. Latin Am J Pharm, 28 (4), 501-6.

Mattos, P. C. (2015). Tipos de Revisão de Literatura. Faculdade de Ciências Agronômicas UNESP, Campus Botucatu.

Moreno, A. H., Nogueira, E. P., Perez, M. P. M. S., \& Lima, L. R. O. (2007). Atenção farmacêutica na prevenção de interações medicamentosas em hipertensos. Rev. Inst. Ciênc. Saúde, 25 (4), 373-7.

Nascimento, D. M. \& Pigoso, A. A. (2013). Interação Medicamentosa entre anti-hipertensivos e anti-inflamatórios não esteroidais. Rev. Cient. FHO Uniararas, 1 (1), 14-17.

Neiva, L. D. B., Oliveira, M. A. C., Neto, M. P. L., Silva, F. V. F., Santos, M. C., Dos Santos, P. N., \& Próspero, D. F. A. (2019). Interações medicamentosas em pacientes nefrológicos em um Hospital de Referência do Nordeste Brasileiro. Revista Eletrônica Acervo Saúde, 29, e949-e949.

Petri, A. A., Schneider, A., Kleibert, K. R. U., Bittencourt, V. L. L., Winkelmann, E. R., \& De Fátima Colet, C. (2020). Interações medicamentosas potenciais em pacientes hospitalizados. Revista de Atenção à Saúde, 18 (63).

Pinto, N. B. F., Vieira, L. B., Pereira, F. M. V., Reis, A. M. M., \& Cassiani, S. H. D. B. (2014). Interações medicamentosas em prescrições de idosos hipertensos: prevalência e significância clínica [Drug interactions in prescriptions for elderly hypertensive patients: prevalence and clinical significance]. Revista Enfermagem UERJ, 22 (6), 735-741.

Pirmohamed, M. (2010). Drug-drug interactions and adverse drug reactions: Separating the wheat from the chaff. Wiener Klinische Wochenschrift, 122 (3-4), 62-64.

Rocha, A. A., Alves, J. D., \& Vitorino, F. R. C. G. (2017). Potenciais interações medicamentosas em pacientes portadores de doença renal crônica em tratamento de hemodiálise. Revista da Universidade Vale do Rio Verde, 15 (2), 112-121.

Santos, T. O. D., Nascimento, M. M. G. D., Nascimento, Y. A., Oliveira, G. C. B. D., Martins, U. C. D. M., Silva, D. F. D., \& Oliveira, D. R. D. (2019). Interações medicamentosas entre idosos acompanhados em serviço de gerenciamento da terapia medicamentosa da Atenção Primária. Einstein (São Paulo), 17 (4).

Scrignoli, C. P., Teixeira, V. C. M. C., \& Leal, D. C. P. (2016). Interações medicamentosas entre drogas mais prescritas em unidade de terapia intensiva adulta. Revista Brasileira de Farmácia Hospitalar e Serviços de Saúde, 7 (2).

Secoli, S.R. (2010). Polifarmácia: interações e reações adversas no uso de medicamentos por idosos. Revista Brasileira de Enfermagem, 63 (1), $136-140$.

Silva, F. R., Canabrava L. B. E., Alves, B. E. L., Araujo, H. F., \& Netto, S. O. (2014). Comparação da evolução das diretrizes de hipertensão norte-americanas nos últimos 17 anos. Rev. Med. Saúde Brasília, 3 (3), 244-52.

Malachias, M. V. B. et al. (2016). $7^{\text {a }}$ Diretriz Brasileira de Hipertensão Arterial. Sociedade Brasileira de Cardiologia. Rio de Janeiro: Arq. Bras. Cardiol., 107 (3), 1-103. 
Research, Society and Development, v. 11, n. 2, e4411225488, 2022

(CC BY 4.0) | ISSN 2525-3409 | DOI: http://dx.doi.org/10.33448/rsd-v11i2.25488

Spanevello, S., Locatelli, C., Bandeira, V. A. C., \& Colet, C. F. (2018). Interações medicamentosas, reações adversas e ajuste de dose de medicamentos utilizados por pacientes em hemodiálise. Saúde (Santa Maria), 3 (44).

Tavares, M. S., Macedo, T. C., \& Guimarães Mendes, D. R. (2013). Possíveis interações medicamentosas em um grupo de hipertenso e diabético da Estratégia Saúde da Família. Revista de Divulgação Científica Sena Aires, 1 (2), 119-125.

Tavares, D. S., Gomes, N. C., Rodriguês, L. R., \& Tavares, D. M. D. S. (2018). Perfil de idosos com síndrome metabólica e fatores associados às possíveis interações medicamentosas. Revista Brasileira de Geriatria e Gerontologia, 21 (2), 164-175.

Zanetti, M. O. B., Marchetti, J. M., \& De Andrade, R. C. G. (2017). Caracterização do perfil de interações medicamentosas potenciais em prescrições da atenção primária de Ribeirão Preto-SP. Revista da Universidade Vale do Rio Verde, 15 (1), 279-288. 